INVESTIGACIÓN

https://doi.org/10.15198/seeci.2020.52.49-72

Recibido: 28/05/2019 --- Aceptado: 17/10/2019 --- Publicado: 15/07/2020

\title{
EL DISCURSO PEDAGÓGICO EN LA ENSEÑANZA DE CONCEPTOS EN QUÍMICA CON EL USO DEL TEXTO ESCOLAR
}

\section{THE PEDAGOGICAL DISCOURSE IN THE TEACHING OF CONCEPTS IN CHEMISTRY WITH THE USE OF THE SCHOOL TEXT}

Pedro A. Certad Villarroel ${ }^{1}$. Universidad Metropolitana. Venezuela. pcertad@unimet.edu.ve

Tulio Ramírez². Universidad Católica Andrés Bello. Venezuela. tuliorc1@gmail.com

\section{RESUMEN}

En el presente estudio se planteó analizar el discurso académico científico en la enseñanza de una selección de conceptos básicos de Química valiéndose del texto escolar como punto de encuentro entre las interacciones. Desde su método pretendió un nivel explicativo bajo un diseño de tipo mixto o combinado con el uso de la técnica de análisis de contenido que sirvió de unión entre datos cuantitativos referidos al conteo de categorías y frecuencia de apariciones dentro del capital verbal con información cualitativa proveniente del registro de interacciones, grabaciones y transcripciones de los hechos observados durante el uso del texto escolar. Al finalizar se pudo concluir que la observación durante las interacciones se caracterizó por el predominio de unidades dialogales frente a las monologales, uso de un lenguaje científico, cercano, centrado, que en algunos momentos era poco preciso e inclusivo. Las secuencias muestran tres momentos diferenciados y una predominancia de la explicación magistral en la cual se hace uso de algunas estrategias con un cierre marcado con la realización de actividades propuestas tanto por el profesor como por el texto escolar. El reconocimiento de modelos de aprendizaje fue muy particular pues en ningún caso se trató de un modelo puro que permitiera enmarcar la clase para así poder identificar cómo sería llevada ésta, qué esperar, qué no esperar, en fin, reconocer la clase y el modelo por el cual era dada; fue a partir del entretejido de los atributos de cada modelo de aprendizaje que se hizo posible caracterizar al profesor.

PALABRAS CLAVE: discurso pedagógico - análisis de contenido - texto escolar conceptos - Química.

1 Pedro A. Certad Villarroel: Facultad de Ciencias y Arte. Departamento de Ciencias de la Educación. Venezuela. pcertad@unimet.edu.ve

2 Tulio Ramírez: Facultad de Humanidades y Educación, Venezuela. tuliorc@gmail.com 


\section{ABSTRACT}

In the present study, it was proposed to analyze the academic scientific discourse in the teaching of a selection of basic concepts of Chemistry using the school text as an encounter point among interactions. From its method it intended an explanatory level under a mixed or combined type design with the use of the content analysis technique that served as a union between quantitative data related to the count of categories and frequency of occurrences within the verbal capital with qualitative information from the record of interactions, recordings and transcripts of the events observed during the use of the school text. At the end it could be concluded that observation during interactions was characterized by the predominance of dialogue units over monologues, using a close focused scientific language, which at times was imprecise and inclusive. The sequences show three different moments and a predominance of the masterful explanation in which some strategies are used with a closing marked by the performance of activities proposed by both: the teacher and the school text. The recognition of learning models was very particular because in no case, it was a pure model that allowed to frame the class in order to identify how it would be carried out, what to expect, what not to expect. In short, to recognize the class and the model by which it was given; it was from the interweaving of the attributes of each learning model that it became possible to characterize the teacher.

KEYWORDS: pedagogical discourse - content analysis - school text - concepts Chemistry.

\section{O DISCURSO PEDAGÓGICO NO ENSINO DE CONCEITOS DE QUÍMICA COM O USO DO LIVRO DE TEXTO}

\section{RESUMO}

No presente estudo foi proposta uma análise do discurso acadêmico científico no ensino de uma seleção de conceitos básicos de Química usando o livro escolar como ponto de encontro entre as interações. A partir da metodologia que pretendía um nível explicativo sob uma estrutura de tipo misto ou combinada com o uso da técnica de análise de conteúdo que funcionou como uma união entre dados quantitativos referidos a contagem de categorias e frequência de aparições dentro do capital verbal com informação qualitativa que vem do registro de interações, gravações e transcrições dos fatos observados durante o uso do texto escolar. Ao finalizar foi possível concluir que a observação durante as interações se caracterizou pelo predomínio de unidades de diálogo frente aos monólogos, uso de linguagem científico, próximo, centrado, que em alguns momentos era impreciso e inclusivo. As sequências mostram três momentos diferenciados e um predomínio da explicação magistral nas quais são feitas algumas estratégias com um fechamento marcado com a realização de atividades propostas tanto pelo professor quanto pelo livro de texto. $O$ reconhecimento de modelos de aprendizado foi muito particular pois em nenhum caso se encontrou um modelo puro que permitisse enquadrar a turma para poder identificar como esta seria tratada, o que esperar, o que não esperar, enfim, reconhecer a turma e o modelo pelo qual a aula era ensinada; foi a partir do 
Certad Villarroel, P. A. y Ramírez, T. El discurso pedagógico en la enseñanza de conceptos en química con el uso del texto escolar

entrelaçado dos atributos de cada modelo de aprendizado que tornou-se possível caracterizar o professor.

PALAVRAS CHAVE: discurso pedagógico - análise de conteúdo - livro de texto conceitos- Química.

\section{Cómo citar el artículo:}

Certad Villarroel, P. A. y Ramírez, T. (2020). El discurso pedagógico en la enseñanza de conceptos en química con el uso del texto escolar. [The pedagogical discourse in the teaching of concepts in chemistry with the use of the school text]. Revista de Comunicación de la SEECI, (52), 49-72.

doi: http://doi.org/10.15198/seeci.2020.52.49-72

Recuperado de http://www.seeci.net/revista/index.php/seeci/article/view/599

\section{INTRODUCCIÓN}

Una clase se nutre en la explicación, entendiendo por explicación la acción que pretende hacer entender un concepto o una situación por medio de una secuencia explicativa (D'Avolio, 2013) también llamada por Zuev (1988) el texto básico, lo cual no es más que una estructura oral y escrita en la que se exponen los conocimientos fundamentales para alcanzar los objetivos propuestos en la enseñanza (D'Avolio, 2013).

Cuando en el aula se produce una explicación, ésta lleva consigo algún tipo de estrategia didáctica e intervienen en ella varios actores como lo son el docente que explica con apoyo tanto en su conocimiento como en el texto escolar, el estudiante que pregunta, atiende, trabaja e investiga en su texto escolar y el texto escolar en sí mismo como vehículo pedagógico. Entre estos actores es que se materializa la explicación general y conceptual, la estrategia de enseñanza y la actividad que promueve el aprendizaje. Es en esta dinámica real en la que se producen las interacciones en el aula para lo que Castro (2007) afirma que:

La interacción es algo que existe una vez que hay dos o más conductas de dos o más sujetos, y son las conductas y algo más, ese algo es el sentido de la relación que hay entre ambas acciones, y tal sentido es la base de la relación que existe entre uno y otro sujeto para un momento o un lapso determinado. (p. 4)

$Y$, en este caso dos o más conductas establecen relación, la del docente y los alumnos con el texto escolar, bien sea de manera individual directa o grupal, y la que se establece dentro del aula con el papel que juegan en ella los participantes.

Como complemento de lo anterior, Bruner (1998) agrega que la actividad ejecutada por el docente tiene lugar en un juego de intercambios, discusiones, diálogos y acuerdos que permiten la puesta en marcha de razonamientos individuales y colectivos, formaciones conceptuales y manejo de materiales donde los contenidos u objetos de aprendizaje deben ser asumidos no como fines en sí mismos, sino como 
insumos para el estímulo de indagaciones y cuestionamientos por parte de los estudiantes.

El rol desempeñado entonces por el docente y por el texto escolar, en el caso de este estudio en la enseñanza de la Química reviste de una capital importancia. Como actores involucrados y como entes discursivos construyen dentro de la clase dinámicas específicas de comunicación, establecen relaciones y a su vez determinan el abordaje de los ejes temáticos por medio de la explicación y de la selección de determinadas estrategias presentadas en el texto escolar, en las cuales se apoya el docente para la planificación y la ejecución de la clase.

En este sentido, desde 1960 se hallan diferentes investigaciones que toman como objeto de estudio la interacción en el aula haciendo centro en la dinámica que se genera en ella (Bellack, 1966; Doyle, 1977) por ejemplo en la formulación de preguntas y respuestas (Barnes, Britton y Rosen, 1971; Sinclair y Couthard, 1975 y Titone, 1986). Desde las posturas pedagógicas ínter estructurante como las de Not (1983) o Perret-Clermont (1984), se señala la necesidad de propiciar estrategias que generen interacciones, donde el alumno pueda participar en esta dinámica de acuerdo con sus características intelectuales, se adecúe el nivel de ayuda a su nivel de competencia y se respeten sus tiempos de construcción cognitiva. Así, la estructura de la tarea académica y el intercambio docente-texto escolar y alumno-texto escolar se alejaría de la marcada intención evaluativa que suele caracterizarse en el chequeo del logro de objetivos eminentemente conductistas que en la búsqueda de actitudes observables dejan por fuera procesos internos de gran valor.

Vygotsky (1979) desde la psicología, fundamenta el lugar que ocupa la mediación en el aprendizaje, en este caso del texto escolar como un vehículo pedagógico. El autor muestra la necesidad de comprender la distancia que debe recorrer el alumno entre lo que ya sabe y lo que puede llegar a saber con ayuda de este mediador, procesos que pueden darse dentro y fuera del aula. En este sentido, Bruner (1998) argumenta que ya no se discute la importancia de la construcción de significados propios por parte del alumno, esta construcción puede llevarse a cabo no solo en la dinámica del aula sino también de manera asincrónica, cuando el estudiante interactúa con el texto o con sus pares fuera del aula.

Más tarde, en la década de los años ochenta del siglo pasado, la preocupación principal de los investigadores en la enseñanza de las ciencias estuvo ligada al análisis de los aprendizajes de los alumnos, a sus concepciones, a la de los materiales didácticos y a las de los docentes. Recién finalizada dicha década se comenzó a relacionar el aprendizaje con el contexto social en el que éste está inmerso, tal como se muestra en los estudios de Fentermacher (1986), en Edwards y Mercer (1987) y en Seeger (1991), contexto que contribuye a los resultados del proceso y que son determinantes como estrategia en la explicación científica cuando se manejan conceptos. De esta forma, se establece de manera implícita la responsabilidad del docente y del texto escolar en el proceso de enseñanza como facilitadores del proceso de aprendizaje del alumno. Estos teóricos han generado 
algunas categorías de análisis que sirvieron de orientación en este estudio para la escogencia de los elementos a observar en la interacción en el aula.

Este artículo se centra entonces en la explicación del docente que con el uso del texto escolar hace para enseñar los conceptos de Medición, Materia y Energía, y toma en cuenta además los aportes de Calsamiglia y Tusón (1999) quienes establecen algunas particularidades que merece la pena atender, entre ellas, la detección de las imprecisiones en la explicación, la imposibilidad de tachar y volver a empezar y la alternativa del docente en este caso en seguir hablando, de allí que los discursos estén teñidos de repeticiones, parafraseos y las florituras o limitaciones que tenga el docente en su esquema comunicacional, aspectos a considerar cuando el profesor hace la explicación y cuyo acto toma como base la explicación existente en el texto escolar.

\section{OBJETIVO}

Para este estudio se planteó como principal objetivo analizar el discurso académico científico que ocurre para la enseñanza de una selección de conceptos básicos de Química cuando el profesor hace uso del uso del texto escolar, y para ello, se ideó un método enmarcado en el paradigma mixto de investigación.

\section{METODOLOGÍA}

El enfoque mixto es un proceso que recolecta, analiza y vincula datos cuantitativos e informaciones cualitativas en un mismo estudio (Mertens, 2009; Tashakkori y Creswell, 2007; Martínez Llantada, 2005; Creswell, 2002; Williams, Unrau, Grinnell y Epstein, 2005 y Tashakkori y Teddlie, 2003) y puede involucrar la conversión de datos cualitativos en cuantitativos y viceversa (Mertens, 2009). Los resultados de este tipo de estudios son en muchos casos numerosos y variados por lo que deben ser ordenados de manera sistemática para interpretar la información recibida. Por este motivo, para el análisis de este tipo de datos se utilizan las triangulaciones, diagramas y programas de informática. Algunos teóricos coinciden en señalar que no existe un único modelo para realizar el análisis de los datos obtenidos y que cada investigador podrá ir perfilando su propio proceso de interpretación. Con base en lo anterior y desde las observaciones de Krippendorff (1980), Miles y Huberman (1984) y Taylor y Bogdan (1986), se decidió establecer el análisis de contenido como la técnica predominante para el abordaje de las observaciones que surgieron de las interacciones.

El análisis de contenido es un procedimiento que permitió analizar y cuantificar los materiales de la comunicación. En general, hace posible analizar con detalle y en profundidad el contenido de una comunicación, sea cual fuere el número de personas y materiales implicados en el proceso, pudiendo emplear cualquier instrumento de compendio de datos como las agendas, diarios, cartas, cuestionarios, encuestas, test proyectivos, libros, anuncios, entrevistas, radio, televisión, entre otras (Holsti, 1968). 
Krippendorff (1980) por su parte, define el análisis de contenido como "la técnica destinada a formular, a partir de ciertos datos, inferencias reproducibles y válidas que puedan aplicarse a un contexto" (p. 28). En el caso de esta investigación, el análisis de contenido permitió la comprensión de los textos escolares en el contexto específico en el cual son generados y usados en interacción con los informantes, además, el manejo del estado del arte y los fundamentos teóricos sobre los cuales se cimentó el estudio. Según Krippendorff (1980) esta técnica sitúa al investigador respecto de la realidad en triple perspectiva:

- Los datos y las informaciones tal y como se comunican al investigador.

- El contexto en el que se generan los datos y las informaciones.

- La forma en que el conocimiento del analista obliga a dividir la realidad para facilitar su comprensión.

El análisis de contenido se configura entonces como una técnica objetiva, sistemática, cualitativa y cuantitativa que trabaja con materiales representativos, marcada por la exhaustividad y con posibilidades de generalización. Para este estudio se aplicó esta técnica a las interacciones entre textos escolares, los conceptos, contextos pedagógicos, los profesores, en fin, a todos integrantes del corpus de investigación.

\subsection{Los textos escolares}

Este parte del corpus está constituido por tres textos escolares usados para la administración de la asignatura Química, en el tercer año de Educación Media General (EMG). El objetivo que llevó a la constitución de este grupo de textos escolares fue recoger una muestra de las explicaciones contenidas en ellos para la enseñanza de Química y realizar un análisis de su uso, manejo conceptual, sus características e implicaciones que pueden tener en el desarrollo del conocimiento.

Con base en lo anterior, se tomó en cuenta para la selección de los textos escolares la frecuencia de uso, la disponibilidad, el perfil de los autores, que fuesen solo libros de teoría y, por último, la edición. A partir de estos criterios de selección, los textos escolares escogidos fueron: Química $3^{\circ}$ año - Serie Conexos de Editorial Santillana. (2013). Ciencia para vivir en comunidad. Ciencias Naturales 3. Tomos 1 y 2 - Colección Bicentenario. (2012), y, Química $3^{\circ}$ año de editorial Romor (2010).

\subsection{Los conceptos}

Para esta parte del corpus se consideró como criterio de selección el tipo de concepto -sistémico, macroconceptos o conceptos supraordenados-, es decir, aquellos que involucran otros grupos de conceptos derivados y dependientes de éste -conceptos básicos y subordinados-. Estos conceptos dan origen a otros en los temas subsiguientes especificados en el programa oficial de la asignatura. Luego, la pertinencia del concepto verificando que los conceptos propuestos estén en el Programa de la Asignatura para el nivel prescrito por tanto son ejes fundamentales en el contenido de los Textos Escolares $y$, por último, la trascendencia escolar 
expresada en el hecho de que su uso reaparezca en los niveles de estudio superiores al que aquí se abordó y en otras asignaturas.

Cumpliendo con los criterios mencionados, los conceptos que integran esta parte del corpus son: Medición, Materia y Energía. Pertenecen al nivel de educación venezolana de tercer $\left(3^{\circ}\right)$ año de EMG; la intención de la escogencia de este nivel de estudio es que es la primera vez que el estudiante se encuentra con la Química como asignatura. Se hace la salvedad que en el programa de Estudios de la Naturaleza correspondiente al primer año de EMG venezolana también se abordan los conceptos de Medición, Materia y Energía, pero desde explicaciones que guardan mayor relación con aspectos de la Física como ciencia.

La aproximación a los tres conceptos se hace desde la lengua y desde Química; más adelante desde la enseñanza, en consecuencia, su trato es combinado: significado lingüístico y definición en Química. Para la selección de estos conceptos se tomó en cuenta que son macroconceptos, es decir, involucran otros grupos conceptuales derivados y dependientes de éste, llamados microconceptos. En segundo lugar, están propuestos en el Programa de la Asignatura en el nivel de educación seleccionado por tanto son ejes fundamentales en el contenido de los Textos Escolares. Por último, su uso reaparece en los programas de la asignatura de los niveles de estudio superiores al que aquí se abordará.

\subsection{El contexto pedagógico}

Para esta parte del corpus se consideraron los planteles educativos. Siendo específicos fueron dieciocho cartas de solicitud de observación que se enviaron a diversos colegios en la zona denominada Gran Caracas, del total de solicitudes hechas, cuatro planteles no tenían docentes en el área de Química por lo tanto no se estaba impartiendo la asignatura, nueve tenían a profesores de otras áreas con conocimientos de Química, tres colegios al no encontrar profesores colocaron a un estudiante avanzado $\mathrm{y}$, por último, solo dos planteles respondieron poder contribuir con el estudio. A continuación, se describen los contextos pedagógicos en los que se llevaron a cabo las observaciones.

\subsubsection{Contexto pedagógico "A"}

Esta institución educativa es de administración pública. Atiende a alumnos y alumnas desde el primero hasta el quinto año de Educación Media General. El Colegio se encuentra ubicado en el Municipio Sucre, de la ciudad de Caracas, Venezuela. El liceo se encuentra en un entorno de clase media-baja perteneciente al estrato IV (Según el método de estratificación desarrollado por Marcel Graffar en Morales Gil, 2003; pp. 56-77). Por otra parte, éste funciona en dos turnos, matutino y vespertino, y en total atiende una matrícula de 818 alumnos. Consta de edificaciones modestas y algo pequeñas. El personal de esta institución está constituido por un Director y dos sub-directores, uno para cada turno, un 
coordinador de seccional, un coordinador de evaluación, un coordinador de control de estudios, dos profesoras orientadoras y cuenta con un total de 31 profesores, de los cuales 11 son del área de Ciencias Naturales.

\subsubsection{Contexto pedagógico "B"}

Este contexto pedagógico es un colegio de administración privada. Atiende a alumnos que van desde los primeros niveles de Educación Inicial hasta el último nivel de EMG. El plantel educativo se encuentra ubicado en el Municipio Sucre, de la ciudad de Caracas, Venezuela. El nivel socio-económico podría denominarse como de "clase media", pertenecientes a los estratos III y IV, de acuerdo a los criterios establecidos en Morales Gil (2003) para el estudio de la población venezolana. De turno matutino, y con actividades complementarias vespertinas. Atiende a una matrícula promedio de 567 estudiantes. Es una edificación de tres pisos, de grandes dimensiones (más de $2000 \mathrm{~m}^{2}$ ). Dispone de 19 aulas de clase, una biblioteca, cinco oficinas administrativas, dos laboratorios, dos expendios de alimentos y baños tanto para el alumnado como para los docentes. Las aulas son ventiladas y amplias con una capacidad aproximada para unos 35 alumnos por curso. El personal de la institución está conformado, por una directora encargada, dos subdirectoras, dos coordinadoras, un coordinador de evaluación, un coordinador de control de estudio, una orientadora y 28 profesores a distintas dedicaciones.

\subsection{Los profesores}

Se consideró necesario abordarlos bien sea por el uso que le dan al texto escolar durante la clase como por la explicación subyacente que proviene de las conceptualizaciones en el texto escolar. En este sentido, el número de profesores de Química a seleccionar fue de tres, debido a que las investigaciones con la tipología acá seleccionada presentan dificultades al momento de compilar la información y al llevar a cabo el análisis de la misma.

Por este motivo, la elección de los informantes se realizó tomando como base los criterios establecidos por Goetz y LeCompte (1988) en cuanto a la selección de los participantes/informantes. Los autores establecen que la selección exige el establecimiento previo de "un conjunto de atributos" (p. 89), en este caso cumplieron con los criterios de administración del curso de Química en el tercer año de EMG, título universitario de profesor de Ciencias Naturales, profesor de Química o licenciatura en Química con componente docente, poseer distintos grados de experiencia, y por último, profesores de diferentes géneros y edades.

Para obtener estas informaciones se aplicó en un primer encuentro la entrevista biográfica, para obtener la información necesaria y pertinente que facilitó la comprensión del marco referencial del que parten sus prácticas pedagógicas específicas. 


\subsubsection{La entrevista biográfica}

Las preguntas de la entrevista biográfica, fueron demográficas, de antecedentes, profesionales y de formación docente. La entrevista se organizó en dos partes: la primera parte en donde se indagan los datos generales de los docentes, y la segunda parte donde se indaga sobre los datos académicos y profesionales de los profesores/informantes.

\subsubsection{Según sus datos generales}

En relación al género se seleccionó un hombre y dos mujeres. La edad se ubicó entre los 34 años y 51 años de edad. En relación al estado civil, el hombre es casado y las dos mujeres son solteras.

Los tres poseían titulación universitaria. En cuanto a los años de experiencia, esta fluctuó entre los 11 y 23 años. Por otro lado, se observó que las dos profesoras cursan estudios de postgrado mientras que el hombre no tiene, ni está cursando estudios de diplomado, especialización, maestría o doctorado.

En relación a la situación laboral, es pertinente señalar que dos de ellos poseen la condición de contratados en la institución en la que laboran, mientras que el tercero está en espera del concurso de oposición que hace el Ministerio para regularizar su presencia en la institución. En lo que tiene que ver con la carga horaria que administra cada profesor, uno de ellos tiene una carga de 28 horas semanales, mientras que los otros dos tienen cargas menores a las 16 horas semanales. Los tres docentes/informantes manifestaron tener otros trabajos en otros contextos pedagógicos.

\subsubsection{Según su perfil académico y profesional}

Aunque los informantes tienen formación en el área, se desempeñan profesionalmente en la educación y están complacidos con su profesión es importante destacar que algunos llegaron a la docencia de manera forzada; todos son docentes activos. Manifestaron que sus formas o estilos de enseñar son diversos y van desde la memorización hasta la construcción del conocimiento. El uso de recursos es diverso, hay manejo de recursos no tecnológicos y tecnológicos. En cuanto al texto escolar, todos los informantes lo usan tanto como apoyo en la explicación como en la asignación de actividades o ejercicios. Consideran importante el trabajo práctico o de laboratorio aspecto que escapa de esta investigación.

\section{ANÁLISIS Y DISCUSIÓN}

Para la sistematización de la experiencia se utilizó la siguiente ficha de recolección que permite integrar las observaciones realizadas en cada sesión de clase (ver tabla $1)$.

Revista de Comunicación de la SEECI. 15 julio, 2020 / 15 noviembre, 2020, n 52, 49-72 
Certad Villarroel, P. A. y Ramírez, T. El discurso pedagógico en la enseñanza de conceptos en química con el uso del texto escolar

Tabla 1. Ficha de recolección de información de las interacciones.

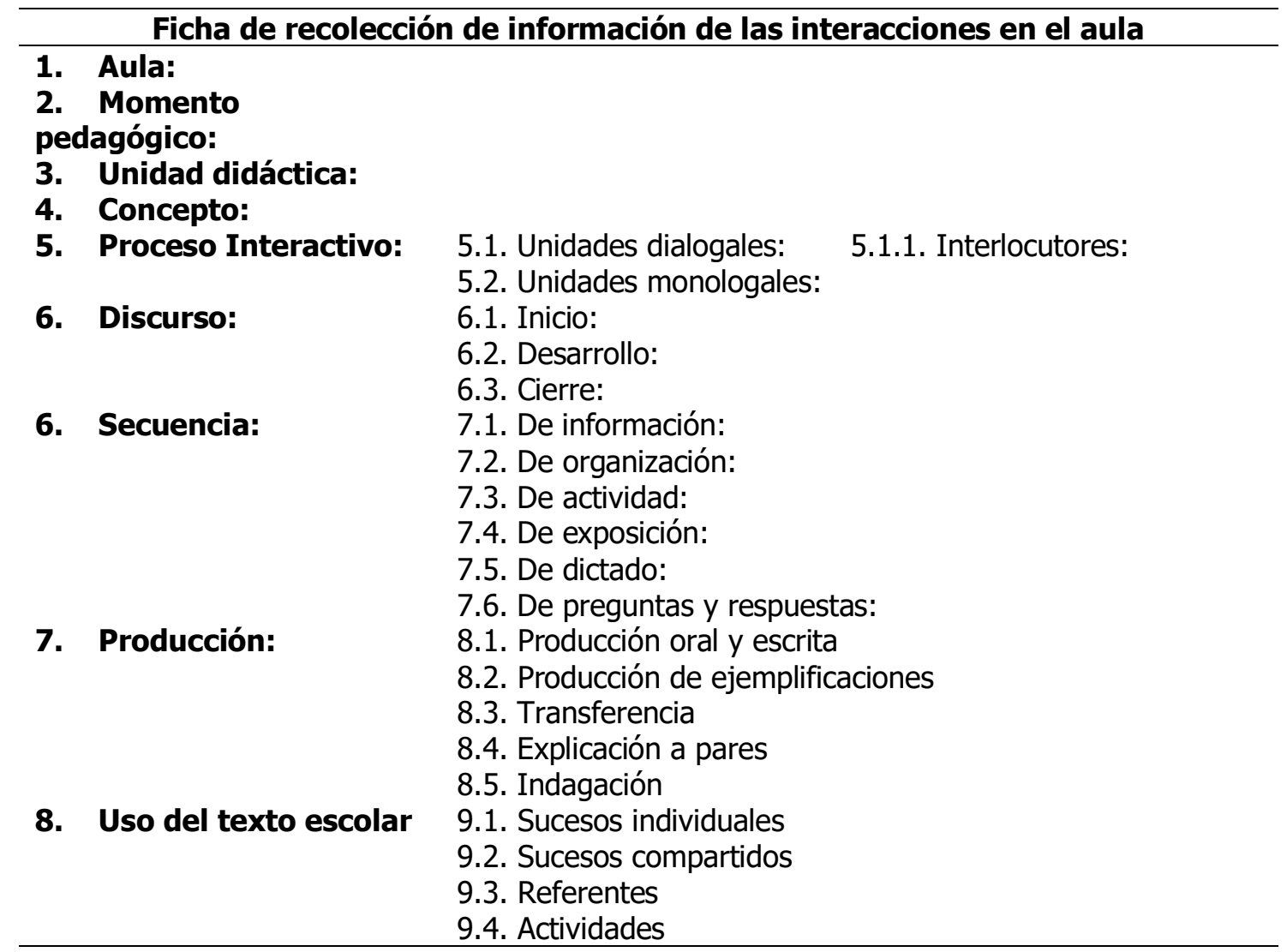

Nota: adaptación de Del Valle (2011).

Fuente: elaboración propia.

\subsection{Profesor/informante "A"}

El aula corresponde al contexto pedagógico " $\mathrm{B}$ " el cual estuvo dividido en dos secciones, una con 24 estudiantes y otra con 25 estudiantes; esta corresponde a la sección de 24 estudiantes. Se realizó la grabación de todas las sesiones que comprendían la unidad didáctica. Las observaciones se realizaron en el primer trimestre del año escolar (período comprendido desde el mes de septiembre hasta el mes de diciembre), intencionalmente seleccionado de acuerdo al contenido administrado y en correspondencia a una planificación que pertenece al plantel educativo y responde al programa oficial de la asignatura (ver tabla 2). Por la variedad de encuentros también es posible observar diversas dinámicas, estados de ánimo, disposiciones al aprendizaje y niveles de cansancio. 
Tabla 2. Ficha de recolección de información de las interacciones en contexto pedagógico " $B$ " Profesor " $A$ ".

\section{Aula: \\ 2. Momento pedagógico \\ 3. Unidad didáctica:}

4. Concepto:

5. Proceso Interactivo:

\section{Discurso:}

\section{Secuencia:}

\section{Producción:}

\section{Ficha de recolección de información de las interacciones en el aula}

\author{
"B" Profesor "A"
}

Primer momento

Los Materiales I: propiedades no características (Texto escolar: Química $3^{\circ}$ año - Editorial Romor. Tema 1.) Medición

5.1. Unidades dialogales: 5.1.1. Interlocutores: Diálogo iniciado por Presentes el profesor y controlado por él usando participación obligatoria.

5.2. Unidades monologales: Presentes

6.1. Inicio: "Buenos días, a ver..."; "silencio"; "saquen el libro y el cuaderno". Se invita a los estudiantes a leer el tema que se tratará en la clase, en silencio y durante 10 minutos. La profesora también realiza la lectura silenciosa.

6.2. Desarrollo: Se hace recapitulación. Con el texto escolar en la mano enuncia "¿grupo, saben lo que es medir?, ¿medición?" "todo lo que nos rodea, la materia, puede ser cuantificada, medida, entonces "¿Qué es la medición?" "¿Qué medimos diariamente?", "¿me siguen?". Acompaña la explicación con señalamientos a objetos dentro del aula y contextualizaciones.

6.3. Cierre: introduce el final con la definición "Entonces medición es el proceso de darle un número y una unidad a la materia para así saber cuánto hay de ella..." Luego, la profesora deja algunos ejercicios copiados en el pizarrón con el título "Asignación-repaso" para que los estudiantes los realicen en casa. En clase subsecuentes no se aborda la resolución de esos ejercicios, sencillamente se continúa con la siguiente parte del tema u otro tema.

7.1. De información: durante el desarrollo de la clase usando situaciones cotidianas: supermercado, abasto, balanza de baño...

7.2. De organización: ofrece turnos de participación sin embargo se dan solapamientos entre los interlocutores lo que se entiende como irrespeto en los turnos del habla.

7.3. De actividad: actividad individual para la resolución de ejercicios copiados en la pizarra y que provienen del profesor. El texto escolar que usa no contiene una sección de actividades al finalizar la unidad temática.

7.4. De exposición: Hecha por el docente y por alumnos

7.5. De dictado: -0-

7.6. De preguntas y respuestas: el profesor realiza preguntas de activación de la discusión, los estudiantes intervienen abiertamente, surgen preguntas de los estudiantes, otros estudiantes responden y el profesor cierra la discusión.

8.1. Producción oral y escrita: La producción que se da es escrita y refiere a la resolución mecánica de ejercicios. No es posible reconocer el manejo adecuado del concepto. En una sesión hubo una aproximación a que los estudiantes hicieran una presentación breve del tema pero la idea quedó diluida y no se ejecutó.

8.2. Producción de ejemplificaciones: -o-

8.3. Transferencia: Durante la participación obligatoria un estudiante explicó cómo era posible hacer una dilución de cloro doméstico (hipoclorito de sodio) utilizando medidas de volumen en casa y la relación que se establece entre la concentración del producto y el poder de limpieza. 
8.4. Explicación a pares: Por intuición los estudiantes se disponen en pares para I resolución de ejercicios. Se hizo notar el dominio de dos estudiantes a la resolución de ejercicios.

8.5. Indagación: -0-

8. Uso del texto escolar 9.1. Sucesos individuales: el texto escolar es usado como un material de lectura durante la secuencia de clase.

9.2. Sucesos compartidos: En algunos momentos, un estudiante lee un fragmento y la clase sigue la lectura en conjunto con la profesora.

9.3. Referentes: El texto escolar es usado como referente continuo en la clase por parte del docente, la clase y la explicación está apoyada en él y acompañado con sus ejemplificaciones.

9.4. Actividades: no contiene.

Fuente: elaboración propia.

La observación en este contexto con este informante se caracterizó por la aparición de unidades dialogales y monologales con un lenguaje coloquial y científico, cercano, con uso de la segunda persona del singular durante la interacción. Se refiere a la clase como "grupo". Continuamente hace alusión a hechos de la vida cotidiana como el pesado de alimentos en establecimientos, medidas del gas doméstico, pesado de personas en farmacias, entre otros, sin embargo, se permean en sus enunciaciones monologales, expresiones de inconformidades políticas, situación económica personal, poder adquisitivo del venezolano antes de 1998 y uso intencional de términos como "enchufados", "tu comandante" y "tenemos patria". Estos términos causan diversas emociones en la clase y permiten ciertas dispersiones dentro de la explicación; el profesor retoma el hilo de la clase y aborda los contenidos con precisión. Define el concepto diciendo:

- (UC1): ...medición es entonces [UR1] 'un proceso, se da por pasos', en el cual el que [UR2] 'mide usando un instrumento adecuado', [UR3-UR4] "otorga una cantidad en número' y una unidad propia de la dimensión que mide a un material'; [UR5] la medida como tal la da el instrumento de medida.

El texto escolar que utiliza este profesor/informante no define expresamente Medición, solo presenta algunas relaciones de este concepto con lo que con él se hace o se logra cotidianamente. A partir de los Libros Especializados de Consulta (LEC), se obtuvo que los atributos que componen este concepto son:

- 'cuantitativo's definida por uso numérico en el concepto.

- 'proceso's definida como un conjunto de fases consecutivas.

- 'exppracexpe' definida como experiencias práticas o experimentos.

- 'unidades! definida por el uso de una cantidad estandarizada de una determinada magnitud, adoptada por convención o por leyes.

- 'uninumero's definida como en binomio número y unidad.

Y, comparando estos atributos con la definición expresada por el profesor/informante en la clase se tuvo que dicha definición presentaba los siguientes atributos:

- 'proceso' "... un proceso, se da por pasos..."

- 'cuantitativo!' "...otorga una cantidad en número..." 
Certad Villarroel, P. A. y Ramírez, T. El discurso pedagógico en la enseñanza de conceptos en química con el uso del texto escolar

- 'uninumero': "...otorga una cantidad en número y una unidad propia de la dimensión que mide a un material..."

Adicionalmente, el profesor/informante desde su experiencia proporcionó un nuevo atributo a la definición como es el instrumento de medida ('instmedida)

- 'instmedida': "...mide usando un instrumento adecuado..." "...la medida como tal la da el instrumento de medida".

En cuanto al proceso interactivo, se observó la presencia de unidades dialogales inducidas y algunas espontáneas las cuales surgieron de la clase con intervenciones que complementaron la explicación del profesor/informante. En cuanto a los marcadores discursivos se dejó ver que el profesor/informante captaba la atención de la clase y aunque los estudiantes hablaban éste no detuvo su explicación. Luego, en las secuencias y durante el desarrollo de la clase el profesor/informante hizo una generación de interrogantes que estimularon la producción de asociaciones con el contexto cotidiano.

Durante el inicio de la clase el profesor/informante solicitó a los estudiantes que sacaran el libro de texto, la clase hace uso de éste y el profesor/informante invitó a su lectura o revisión, en todo momento lo mantuvo en su mano o sobre la mesa y en el tema en estudio. Este texto escolar, por su construcción particular no fue utilizado para la asignación de actividades de resolución de ejercicios -cálculos matemáticosrespecto a este concepto. La evaluación que se realizó para verificar el aprendizaje de la definición y manejo del concepto quedó ceñido a una repetición memorística en la primera parte de una prueba escrita objetiva, en donde también se pidió el reconocimiento de algunos instrumentos de medición; ambos ítems tuvieron un promedio de 1,34 puntos sobre 2 puntos. Los ejercicios que se realizaron en el aula fueron parte de la evaluación formativa y por cuestiones de tiempo no se les indicó a los estudiantes si sus resultados eran correctos o incorrectos.

Posterior a revisar las grabaciones y las anotaciones hechas durante los encuentros con este profesor/informante, fue posible afirmar que las actitudes mostradas por los estudiantes durante el desarrollo de las clases eran de disposición a atender y a intervenir. La dinámica discursiva áulica se dio en un carácter horizontal, dirigida por el profesor/informante hacia la asociación rústica del concepto con su utilidad y aplicación en el contexto próximo del estudiante.

\subsection{Profesor/informante "B"}

Esta aula corresponde al contexto pedagógico " $\mathrm{A}$ " conformado por 59 estudiantes. Se realizó la grabación de todas las sesiones que comprendían la unidad didáctica. Las observaciones se realizaron en el primer momento del año escolar (período comprendido desde el mes de septiembre hasta el mes de diciembre) intencionalmente seleccionado de acuerdo con el contenido administrado y de acuerdo a una planificación instruccional que corresponde tanto al plantel educativo como al programa oficial de la asignatura (ver tabla 3). Por la variedad de 
encuentros también es posible observar diversas dinámicas, estados de ánimo, disposiciones al aprendizaje y niveles de cansancio.

Tabla 3. Ficha de recolección de información de las interacciones en contexto pedagógico " $A$ " Profesor " $B$ ".

1. Aula:

2. Momento pedagógico:

3. Unidad didáctica:

4. Concepto:

5. Proceso Interactivo:

6. Discurso:

\section{Secuencia:}

\section{Producción:}

\section{ción de información de las interacciones en el aula}

"A" Profesor "B"

Primer momento

Los Materiales I: propiedades no características (Ciencia para vivir en comunidad. Ciencias Naturales 3. Tomo 1. Tema 8 - Colección Bicentenario)

Materia.

5.1. Unidades dialogales: presentes.

5.1.1. Interlocutores: Diálogo iniciado por el profesor y controlado por él usando participación obligatoria.

5.2. Unidades monologales: $X$ en predominancia.

6.1. Inicio: "bueno, bueno..."; "presten atención"; "saquen el libro". Este tiempo fue prolongado y ameritó alzas del tono de voz para lograr el silencio, pero no la atención. La mirada de los estudiantes era dispersa.

6.2. Desarrollo: "vamos a ver quién puede definir materia" "¿Qué es materia?", "¿Qué no es materia entonces...?" Siendo que acá inicia la explicación, aunque hace preguntas dirige sus respuestas a alumnos específicos y ante el silencio o negativa de respuesta no hace orientaciones que conlleven a dar una aproximación a la respuesta que en ocasiones dista de la verdadera explicación científica; inmediatamente él mismo responde. Durante este desarrollo también se llevan a cabo actividades reflexivas pero con las misma dinámica que con las preguntas abiertas de indagación. 6.3. Cierre: "En resumen entonces..." "copien...". El cierre quedó sumido al dictado de la definición que hizo el docente, con libro en mano, pero que sacó de su memoria.

7.1. De información: durante el inicio de la clase a manera de relación con conocimiento previo, específicamente con la unidad didáctica anterior en donde se abordó la medición, escalas de medida e instrumentos de medición. Se infiera que durante esa clase hicieron mediciones a diversos materiales de gran tamaño. 7.2. De organización: El orden de la palabra no es aleatorio ni espontáneo, son designaciones de participación a dedo.

7.3. De actividad: Aunque se proponen actividades éstas no son realizadas por los estudiantes sino por el mismo profesor.

7.4. De exposición: En la exposición predomina la clase magistral hecha por el docente-.

7.5. De dictado: para copiar la definición enunciada por el docente en el cuaderno de los estudiantes al finalizar la clase.

7.6. De preguntas y respuestas: el profesor realiza preguntas de activación de la discusión, asigna interlocutores para responder, responde lo que él mismo ha preguntado, surgen preguntas de los estudiantes, otros estudiantes responden y el profesor cierra la secuencia.

8.1. Producción oral y escrita: -o-

8.2. Producción de ejemplificaciones: -o-

8.3. Transferencia: -o-

8.4. Explicación a pares: -o-

8.5. Indagación:-o- 
Certad Villarroel, P. A. y Ramírez, T. El discurso pedagógico en la enseñanza de conceptos en química con el uso del texto escolar

\begin{tabular}{ll}
\hline 9. Uso del texto escolar & 9.1. Sucesos individuales: el texto escolar es usado como material \\
de lectura durante la clase. \\
9.2. Sucesos compartidos: -o- \\
9.3. Referentes: -o- \\
9.4. Actividades: solo las actividades de tipo reflexivas que no \\
llegan a concluirse ni a evidenciar algún tipo de aprendizaje.
\end{tabular}

Fuente: elaboración propia.

El docente tuvo dificultad para iniciar las intervenciones de los estudiantes, fue necesario una facecia para dar pie a las intervenciones. El docente utiliza un lenguaje cercano a los estudiantes y los involucra en la discusión haciendo uso del tuteo. Se deja ver una estructura en su explicación. Una vez definido el concepto lo relaciona mediante el uso de ejemplos traídos del contexto.

El profesor/informante definió el concepto diciendo:

- (UC2): ...materia es todo los que nos rodea, [UR6] 'todo lo que ocupa un lugar en el espacio' y [UR7] 'posee una masa'. Por ejemplo, las mesas son materia, el piso es materia, esa fruta es materia, el termo es matera, todo, todo lo que nos rodea es materia. ¿Qué más es materia?, ¿Qué no es materia entonces...? Dime -señalando a un estudiante-.

El texto escolar que utilizó este profesor/informante define Materia diciendo:

- (UC3): "Piensa por un momento en las cosas materiales que tienes en casa, encontrarás: aparatos electrodomésticos, muebles, camas, lámparas, mesas. Estos objetos son perceptibles a simple vista, tienen una masa determinada y ocupan un lugar en el espacio, en sentido general los llamamos Materia" (MPPE, 2012; p. 148).

Tanto los LEC como el texto escolar utilizado por este profesor/informante coinciden en que la constitución de este concepto viene dada por las siguientes categorías:

- 'tenencia!: "...tienen una masa determinada..."

- 'ocupación! "...ocupan un lugar en el espacio..."

- 'percepción': "...son perceptibles a simple vista..."

Comparando estos atributos con la definición expresada por el profesor/informante en la clase se tiene que éste deja por fuera los aspectos asociados a la percepción sensorial. Su definición empleada en aula presenta los siguientes atributos:

- 'tenencia' "... y posee una masa..."

- 'ocupación! "...todo lo que ocupa un lugar en el espacio..."

Durante la interacción, se evidenció la presencia de unidades dialogales de manera forzada seleccionando quién debe responder. En cuanto a los marcadores discursivos se reconoció que en el inicio de la clase se captaba la atención mediante un alza del tono de voz para llamar la atención del grupo. Luego, durante el desarrollo se hizo una generación de interrogantes, pero aludiendo a

Revista de Comunicación de la SEECI. 15 julio, 2020 / 15 noviembre, 2020, nº 52, 49-72 
aspectos memorísticos más que al análisis o a la aproximación al contexto. Sin haber respuesta por parte de los estudiantes, el profesor/informante conceptualizó y de una manera emocionada comenzó a nombrar situaciones que referían al concepto. Esta emoción hizo que los estudiantes le miraran y se centraran en su discurso, además, movía los brazos y señalaba muchos objetos presentes en el aula de clase, y de una manera impulsiva nombró muchos diciendo:

- (UC4): Este concepto debe quedar muy claro, deben aprenderlo, de él derivan muchos otros conceptos como el que sigue que es medición, porque la materia debemos medirla.

Su lenguaje, aunque científico, está dirigido a un estudiante en formación y es preciso, sin embargo, intentó conectar el concepto de Materia con átomo y se hizo necesario hacer un grupo de explicaciones que quizás no estaban indicadas en la unidad didáctica. Cuando esto sucedió se dio la siguiente imprecisión:

- (UC5): "El átomo es la partícula más pequeña que existe".

Si se analiza lo que el profesor/informante mencionó, se obtiene que:

- El átomo es definido en el campo de la Física y de la Química como la cantidad menor de un elemento químico que tiene existencia propia y que se consideró indivisible. Se compone de un núcleo, con protones y neutrones, y de electrones orbitales, en número característico para cada elemento químico (RAE, 2001; Mahan y Myers, 1990; Whitten, Galley y Davis, 1992).

- El átomo está constituido por un núcleo denso con carga positiva que se encuentra rodeado por electrones con carga negativa relativamente lejanos. El núcleo está constituido por partículas subatómicas llamadas neutrones, las cuales son eléctricamente neutras, y protones, que tienen carga positiva (McMurry, 2001; Mahan y Myers, 1990; Whitten, Galley y Davis, 1992 y Ebbing, 1997).

Por lo tanto, el átomo es la cantidad menor de un elemento químico, el cual está constituido por otras partículas, pero existen partículas más pequeñas que el átomo mismo las cuales lo constituyen.

Otra imprecisión se observó cuando, durante la explicación, el profesor aludió a objetos visibles diciendo:

- (UC6): las mesas son materia, el piso es materia, esa fruta es materia, el termo es materia...

Pero no lo hace a estados físicos gaseosos los cuales también son Materia.

Aunque durante el inicio de la clase el profesor/informante solicitó a los estudiantes que sacaran el libro de texto, él lo mantuvo sobre la mesa sin hacer uso de éste, solo refirió al texto escolar para la definición del concepto e invitó a su lectura o revisión. Se plantearon actividades del tipo reflexiva, leídas y respondidas por el profesor. Los estudiantes hacían lectura del texto en la medida que la explicación del docente se daba y las intervenciones eran aisladas predominando una 
actitud general de tipo pasiva. La evaluación que se realizó para verificar el aprendizaje de la definición y manejo del concepto se centró en la repetición memorística de la definición en una prueba escrita objetiva en donde también se propusieron dos ejercicios de cálculo de densidad como la relación establecida entre la masa y el volumen de un material; los ítems tuvieron un promedio de 2,11 puntos sobre 4 puntos. La imprecisión del átomo se mostró en respuestas de la definición del concepto y quedó sin especificar si el estado gaseoso es materia o no lo era.

Luego de revisar las grabaciones y las anotaciones hechas durante los encuentros con este profesor/informante, fue posible afirmar que las actitudes mostradas por los estudiantes durante el desarrollo de las clases eran de disposición a atender, pero no a intervenir. Las intervenciones puntuales no generaron argumentaciones sino confirmaciones de la extensión de la definición. La dinámica discursiva áulica se dio en un carácter horizontal, dominado por el profesor/informante, fundamentado en la segunda persona del singular y retórica dirigida por la intimidación hasta la memorización de la definición.

\subsection{Profesor/informante " $C$ "}

Esta aula corresponde al contexto pedagógico " $\mathrm{B}$ " el cual estuvo dividido en dos secciones, una con 24 estudiantes y otra con 25 estudiantes y esta aula corresponde a la sección de 25 estudiantes. Se realizó la grabación de todas las sesiones que comprendían la unidad didáctica. Las observaciones se realizaron en el segundo momento del año escolar (período comprendido desde el mes de enero hasta el mes de abril) intencionalmente seleccionado de acuerdo al contenido administrado y a su planificación instruccional (ver tabla 4). Por la variedad de encuentros también fue posible observar diversas dinámicas, estados de ánimo, disposiciones al aprendizaje y niveles de cansancio.

Tabla 4. Ficha de recolección de información de las interacciones en el contexto pedagógico " $B$ " Profesor " $C$ ".

\section{Aula:}

2. Momento pedagógico:

3. Unidad didáctica:

4. Concepto:

5. Proceso Interactivo:

6. Discurso:

\section{Ficha de recolección de información de las interacciones en el aula}

"B" Profesor "C"

Segundo momento

Unidad III: La Estructura de la Materia. Tema 2: La teoría atómica. Química $3^{\circ}$ año - Serie Conexos - Editorial Santillana

$$
\text { Medición: Materia: Energía: }
$$

5.1. Unidades dialogales: $\mathrm{X}$

5.1.1. Interlocutores: Diálogo iniciado por el profesor y controlado por él usando participación obligatoria.

\subsection{Unidades monologales:}

6.1. Inicio: "Que calor hace ¿verdad?"; "¿por qué hará tanto calor?"; "cómo llegará ese calor hasta acá?"

6.2. Desarrollo: "ese calor que está haciendo es una forma de energía, la luz que entra por la ventana o si golpeamos la mesa y se produce un sonido... ahí hay energía." "en base a estas observaciones ¿podremos definir energía?".

6.3. Cierre: "para finalizar entonces, rápidamente, diez de ustedes que compartan un ejemplo de energía que observen en su entorno cotidiano." 


\section{Secuencia:}

\section{Producción:}

\section{Uso del texto escolar}
7.1. De información: durante el inicio de la clase a manera de interpelaciones con situaciones cotidianas.
7.2. De organización: participaciones espontáneas por parte de los estudiantes para establecer significados.
7.3. De actividad: para el cierre de la clase en la que se pretende el crecimiento de relaciones entre el concepto y el hecho cotidiano.

7.4. De exposición: Hecha por el docente

7.5. De dictado: No se observó.

7.6. De preguntas y respuestas: el profesor realiza preguntas de activación de la discusión, los interlocutores surgen ordenadamente para responder, hay preguntas de los estudiantes, otros estudiantes responden y el profesor cierra la secuencia.

8.1. Producción oral y escrita: Tanto la producción oral como la escrita se dan durante la clase, así como la lectura e intercambio de los productos. Esta dinámica posibilita la evaluación continua de los procesos de construcción conceptual, ejemplos, contextualizaciones e indagación.

8.2. Producción de ejemplificaciones: La mayoría de clase es capaz de proponer ejemplos que surgen de situaciones vividas y de situaciones que están ocurriendo durante la clase. Las ejemplificaciones estuvieron cónsonas con los atributos del concepto.

8.3. Transferencia: Cuando se abordan los distintos tipos de Energía, los estudiantes reconocieron en cada uno tanto el trabajo como el calor, dos conceptos atributos manejados en temas previos a este de Energía. Esto evidencia la habilidad de poder transferir de manera positiva y lateral.

8.4. Explicación a pares: Durante la resolución de las actividades los estudiantes se dispusieron en parejas por instrucción de la profesora y se pudo observar que ambos estudiantes participaban en la actividad prestándose apoyo y adarando dudas por igual. 8.5. Indagación: Se produjo de manera espontánea con la intención de buscar otros tipos de Energía además de las que estaban presentes en el texto escolar.

9.1. Sucesos individuales: -o-

9.2. Sucesos compartidos: se lleva a cabo para la aproximación al concepto, como complemento de la secuencia explicativa del docente.

9.3. Referentes: Durante clase, el texto escolar sirve de soporte a la explicación y a las actividades.

9.4. Actividades: Se discuten las actividades de indagación, se profundiza en la activación de hipervínculos por medio de herramientas tecnológicas como tabletas y teléfonos móviles y, por último, se realizan y comparten las actividades finales de verificación.

\section{Fuente: elaboración propia.}

Se observó la existencia de unidades dialogales con un lenguaje científico acorde al nivel que se aborda, con el uso de significados adecuados y de transposiciones didácticas claras y correspondientes al concepto a enseñar; el diálogo se hace en primera, segunda y tercera persona del singular con uso de los nombres propios de cada uno de los estudiantes, sin embargo, cuando éstos quieren dirigirse a la docente lo hacen llamándola "profesora". Continuamente se alude a sucesos o hechos de la vida cotidiana para establecer relaciones entre el concepto y un hecho

Revista de Comunicación de la SEECI. 15 julio, 2020 / 15 noviembre, 2020, nº 52, 49-72 
palpable que demuestre la existencia, características y diversos tipos de Energía. La clase se mantiene enmarcada en el hecho científico, no se hacen alusiones a otro tema que no tenga que ver directamente con el eje que se desarrolla en la clase. El profesor/informante definió el concepto de Energía como:

- (UC7) ...es un concepto complejo porque muchas veces no logramos verla, pero si nos es fácil sentirla. La Energía es [UR8] 'una fuerza que existe en el ambiente, que puede generar cambios, que puede cambiar' y [UR9] 'que en este proceso genera trabajo', pero no un trabajo como el que hacemos regularmente como tarea, no, de ese no, un trabajo mecánico, de cambio en la materia.

El texto escolar que utiliza este profesor/informante no define de manera explícita el concepto de Energía. Como se mencionó anteriormente, aunque el texto escolar hace uso del concepto de Energía pareciera que presupone de forma pragmática su manejo por parte del alumno y esto se muestra en el abordaje de situaciones referidas al concepto de átomo en modelos atómicos y en naturaleza de la Materia donde aborda los tipos de Energía, el comportamiento de partículas, saltos electrónicos entre otros. De acuerdo a los LEC, los atributos que conforman este concepto son:

- 'capacidad's definida como propiedad de la energía de hacer

- 'capacidad1's definida como trabajo

- 'capacidad2': definida como calor

Comparando estos atributos con definición expresada por el profesor/informante durante la clase se tiene que, dicha definición presenta:

- 'capacidad': "...es una fuerza que existe en el ambiente, que puede generar cambios, que puede cambiar..."

- 'capacidad1' "...que en este proceso genera trabajo..."

A partir de esta definición surgen unidades dialogales espontáneas las cuales emergen de la clase. Estas unidades son ejemplos de los estudiantes. En cuanto a los marcadores discursivos se deja ver que el profesor/informante captaba la atención de la clase, no hay teléfonos móviles en uso ni otros dispositivos electrónicos, la audiencia está en atención a la profesora.

El profesor/informante sugirió el uso del libro de texto justo antes de la definir el concepto y lo hizo para mostrar imágenes que complementaran la explicación. No refirió a él para la definición ni se invitó a realizar una lectura de éste solo como apoyo gráfico. Las actividades fueron realizadas en el aula en el cual la profesora indicó el trabajo en parejas. Los estudiantes seleccionaron sus parejas sin inconveniente en un clima cordial. Las actividades se realizaron en el orden que presenta el texto escolar y se discutieron en pleno las dudas que surgían y en la mayoría de los casos los mismos estudiantes respondían las inquietudes de los compañeros y, de ser necesario, la profesora intervenía. La evaluación que se realizó para verificar el aprendizaje de la definición y manejo del concepto fue por medio del reconocimiento de algunos tipos de Energía presentes en imágenes, sus posibles transformaciones y la redacción de un párrafo en el cual es estudiante hiciera una 
producción sobre la Energía en su contexto inmediato; en esta evaluación el curso tuvo un promedio de 1,60 puntos sobre 2 puntos.

Por último, luego de revisar las grabaciones y las anotaciones hechas durante los encuentros con este profesor/informante, se afirmó que la dinámica establecida en la clase fue bastante particular pues promovió la intervención de los estudiantes de manera espontánea, atentos, proactivos y dispuestos a expresar sus ideas y acuerdos de la temática compartida; todo esto formó parte de la evaluación formativa de los estudiantes. Los aspectos discursivos se dieron de manera horizontal y respetuosa, orientado por el profesor/informante hacia la asociación del concepto con fenómenos producidos en el contexto del estudiante.

\section{CONCLUSIONES}

Tal como se mostró en los análisis individuales, la observación se caracterizó por el predominio de unidades dialogales ante las monologales con el uso de un lenguaje científico, cercano, centrado, en algunos momentos poco preciso e inclusivo. Las secuencias en el aula muestran tres momentos diferenciados, un inicio en la cual se aplica la recapitulación inducida o provocada por los profesores y que se presenta por la aplicación estratégica de preguntas de activación y focalizadores que intentan conectar conocimientos previos con aquellos que se abordarán en la clase. Luego la aparición de un marcador lingüístico y a partir de él se inicia la explicación central de la sesión, con una predominancia en la explicación magistral en la cual se aplicaron algunas estrategias didácticas y, por último, el cierre marcado con la realización de actividades.

Se denota el uso de la contextualización (Izquierdo Aymerich, 2004) con intenciones similares a las encontradas en los textos escolares, pero con una frecuencia de aparición mucho mayor $(f=11)$; de hecho, es la estrategia didáctica más reconocida en las interacciones lo que lleva a pensar que el profesor sabe su potencialidad al momento de la explicación en Química, criterio que puede provenir de su formación académica y que también se muestra en Ros (2011), Meroni, Copello, y Paredes (2015) y por último en Moraga Toledo, Espinet Blanch y Merino Rubilar, (2019)

Los docentes que conformaron las observaciones egresaron de universidades reconocidas y cada una con un énfasis particular en un modelo de enseñanza, es decir, unos profesores que aprendieron por medio de la aplicación del modelo conductista, otro con el modelo cognoscitivista y algunos con el modelo constructivista; con al menos quince años de graduados, unos con estudios de postgrado o actualización docente, y todos con una visión particular hacia la enseñanza de Química.

El reconocimiento de modelos de aprendizaje en las clases observadas fue muy particular y se dificultó la identificación de un modelo puro que pudiera enmarcar la clase y así poder identificar cómo sería llevada ésta - al igual que en Cordero (2019) -, qué esperar, qué no esperar para poder así reconocer la clase y el modelo por el 
cual era dada, pero, como si estaban claros los atributos de cada modelo de aprendizaje, se pudo caracterizar al profesor por su manera de enseñar.

El primer caracterizado fue el Profesor-predicador. Esta caracterización en algo curiosa. Se trata de un docente que requiere un ambón para hablar y no abandona el texto escolar de su mano, abierto en la página que apoya su explicación. Su contextualización ocurre desde su vivencia personal e interpela a la clase hurgando en sus recuerdos sin dejar de lado el tinte político que afecta su entorno. La interacción que hace es particular, en un primer momento es protagonista, pero luego que se activan las condiciones pasa a espectador y la clase es la que interviene. Recita las definiciones como una oración aprendida por lo que la memorización tiene un papel importante en su proceso de enseñanza.

El segundo caracterizado fue el Profesor-catedrático. Una tipología de académico que vierte hacia sus alumnos una clase de estructura rígida, con una detallada explicación y un libro de texto que queda de adorno sobre el escritorio. Sus clases son magistrales, con una predominancia en el monólogo en el cual los estudiantes no tienen mayor participación pues propicia solo su participación. Es el tipo de docente tradicional, conservador y protagonista. Su preocupación se centra en tres aspectos, en ofrecer información a los estudiantes, en su memoria y en la de los estudiantes. Se traza un objetivo y se encarga de trasmitir los contenidos que se propone, no se interesa por la motivación y hasta se olvida en cierta forma de la dimensión humana del estudiante.

El tercer caracteriza es el Profesor-detallista. Esta tipología corresponde a lo que parece el ideal. Tiene claro desde antes de entrar a clase lo que hará, todo está pensado para que los estudiantes aprendan, inamovible en el propósito de su explicación. No se conforma con lo tradicional que, aunque sea bueno podría ser mejor, por ello trae alguna actividad novedosa, algo que rompa los esquemas. Su explicación es inobjetable, el texto escolar es el libro de apoyo y exprime cada aparte, desde la presentación hasta las actividades finales. Su trato con los estudiantes es horizontal y de respeto, promueve la educación de los alumnos y los entretiene. Su centro es promover la confianza en los alumnos para llevar a cabo la indagación y la intervención espontánea.

Por último, en cuanto al manejo conceptual es posible concluir que se le da mayor importancia y dedicación al aprendizaje de definiciones que pudieran verse como aisladas que a la enseñanza de conceptos, lo que se confirma en las estrategias evaluativas (Certad, 2016). La mayoría de los profesores/informantes le dan relevancia a la memorización, al "caletre" de las definiciones más que a la comprensión del fenómeno químico, se les dictan, y se le pide que las repitan buscando que sean aprendidas.

\section{REFERENCIAS BIBLIOGRÁFICAS}

Barnes, D. R., Britton, J. N. \& Rosen, H. (1971). Language, the Learner and the School. Penguin Books.

Revista de Comunicación de la SEECI. 15 julio, 2020 / 15 noviembre, 2020, n 52, 49-72 
Certad Villarroel, P. A. y Ramírez, T. El discurso pedagógico en la enseñanza de conceptos en química con el uso del texto escolar

Bellack, A. A., Kliebard, H. M., Hyman, R. T. \& Smith, F. L. Jr. (1966). The Language of the Classroom. New York: Teachers College Press, Columbia University.

Bruner, J. (1998). Realidad mental y mundos posibles. Barcelona: Gedisa.

Calsamiglia, H. y Tusón, A. (1999). Las cosas del decir. Barcelona: Ariel.

Castro, A. V. (2007). Un sistema para el análisis de la interacción en el aula. Revista Iberoamericana de Educación, 42(3), 1-12.

Certad, P. A. (2016). El manejo conceptual en química a través de redes sociales. Vivat Academia, (134), 1-21.

Cordero, S. (2019). Modelos didácticos personales de profesoras noveles de biología. En $V$ Jornadas de Enseñanza e Investigación Educativa en el campo de las Ciencias Exactas y Naturales 8 al 10 de mayo de 2019 Ensenada, Argentina. Universidad Nacional de La Plata. Facultad de Humanidades y Ciencias de la Educación. Departamento de Ciencias Exactas y Naturales.

Creswell, J. W. (2002). Educational research: Planning, conducting, and evaluating quantitative (pp. 146-166). Upper Saddle River, NJ: Prentice Hall.

D'Avolio, C. (2013). La representación de las prácticas sociales y discursivas en los textos escolares venezolanos: el problema de los géneros discursivos. (Trabajo de ascenso inédito). El Mácaro, Turmero, Venezuela: Universidad Pedagógica Experimental Libertador, I. P. R.

Del Valle, M. (2011). El análisis del discurso en los textos escolares de Historia. (Tesis Doctoral). Venezuela: Universidad Pedagógica Experimental Libertador.

Doyle, W. (1977). Learning the classroom environment: an ecological analysis. Journal of Teacher Education, 28(6), 51-55.

Ebbing, D. D. (1997). Química General. McGraw-Hill: México.

Edwards, D. y Mercer, H. (1988). El conocimiento compartido: El desarrollo de la comprensión en el aula. Barcelona: Paidós-MEC.

Fentermacher, G. (1986). Tres aspectos de la filosofía de la investigación sobre la enseñanza. Planeta. Caracas. Venezuela.

Goetz, G. \& LeCompte, M. D. (1988). Etnografía y diseños cualitativos en investigación etnográfica. Madrid, España: Morata

Holsti, O. R. (1968). Content analysis. The handbook of social psychology, (2), 596692.

Revista de Comunicación de la SEECI. 15 julio, 2020 / 15 noviembre, 2020, nº 52, 49-72 
Certad Villarroel, P. A. y Ramírez, T. El discurso pedagógico en la enseñanza de conceptos en química con el uso del texto escolar

Izquierdo Aymerich, M. (2004). Un nuevo enfoque de la enseñanza de la química: contextualizar y modernizar. En Anales de la Asociación Química Argentina, 92(4$6), 115-136$.

Krippendorff, K. (1980). Content analysis an introduction to its Methodology. London: Sage.

Mahan, B. C. y Myers, R. J. (1990) Curso de Química Universitario. México: Editorial Addison-Wesley Iberoamericana.

Martínez Llantada, M. A. (2005). Los métodos de investigación educacional: lo cuantitativo y lo cualitativo. En Metodología de la Investigación Educacional. Desafíos y polémicas actuales, (pp. 85-95). La Habana: Pueblo y Educación.

McMurry, J., Mondragón, C. H. y Pozo, V. G. (2001). Química orgánica. International Thomson.

Meroni, G., Copello, M. I. y Paredes, J. (2015). Enseñar química en contexto. Una dimensión de la innovación didáctica en educación secundaria. Educación química, 26(4), 275-280.

Mertens, D. (2009). Research and evaluation in education and psychology: Integrating diversity with quantitative, qualitative, and mixed methods. Thousand Oak: Sage.

Miles, M. \& Huberman, A. (1984) Qualitative Data Analysis. A sourcebook of New Methods. California, Estados Unidos: Sage.

Moraga Toledo, S., Espinet Blanch, M. y Merino Rubilar, C. (2019). El contexto en la enseñanza de la química: Análisis de secuencias de enseñanza y aprendizaje diseñadas por profesores de ciencias de secundaria en formación inicial. Revista Eureka sobre Enseñanza y Divulgación de las Ciencias, 16(1).

Morales Gil, E. (2003). La exclusión de los pobres de la educación superior venezolana. Caracas: Ministerio de Educación Superior.

Not, L. (1983). Las pedagogías del conocimiento. (2a. Ed.). México: Fondo de Cultura Económica.

Perret-Clermont, A. (1984). La construcción de la inteligencia en la interacción social: aprendiendo con los compañeros. Madrid: Aprendizaje Visor.

Ros, A. C. (2011). Enseñar química mediante la contextualización, la indagación y la modelización. Alambique: Didáctica de las ciencias experimentales, (69), 21-34.

Seeger, A. (1991). Styles of musical ethnography. Comparative Musicology and Anthropology of Music: Essays on the History of Ethnomusicology, 342-55. 
Certad Villarroel, P. A. y Ramírez, T. El discurso pedagógico en la enseñanza de conceptos en química con el uso del texto escolar

Sinclair, J. M. \& Coulthard, M. (1975). Towards an analysis of discourse: The English used by teachers and pupils. Oxford University.

Tashakkori, A. \& Creswell, J. W. (2007). The New Era of Mixed Methods. Journal of Mixed Methods Research, 1(1), 3-7. doi: https://doi.org/10.1177/2345678906293042

Taylor, S y Bogdan, R. (1986) Introducción a los métodos cualitativos de Investigación: La búsqueda de significados. Buenos Aires, Argentina: Paidós.

Tashakkori, A. \& Teddlie, C. (2003). Handbook of Mixed Methods in Social \& Behavioral Research. Thousand Oaks: Sage.

Titone, R. (1981). Psicodidáctica (Vol. 20). Narcea Ediciones.

Vygotsky, L. S. (1979). El desarrollo de los procesos psicológicos superiores. Barcelona: Crítica.

Whitten, K. D., Galley, R. E. y Davis, D. (1992). Química General. Mc Graw Hill: México.

Williams, M., Unrau, Y. A., Grinnell, R. M. \& Epstein, I. (2005). The qualitative research approach. Social work research and evaluation: Quantitative and qualitative approaches, (7), 75-87.

Zuev, D. (1988). El libro de texto. La Habana: Pueblo y Educación.

\section{AUTORES:}

\section{Pedro A. Certad Villarroel}

Lic. en Educación, especialista en Tecnología, Aprendizaje y Conocimiento, Doctor en Educación (UCV). Profesor asociado en la Universidad Metropolitana, Profesor investigador, Director para el desarrollo de competencias y Director (E) de estudios iniciales en la Universidad Metropolitana. Universidad de adscripción: Universidad Metropolitana (UNIMET).

pcertad@unimet.edu.ve

Orcid ID: https://orcid.org/0000-0002-5936-834X

ResearchGate: https://www.researchgate.net/profile/Pedro Certad

\section{Tulio Ramírez}

Abogado, Sociólogo, MSc. en Formación de Recursos Humanos, Doctor en Filosofía y Ciencias de la Educación (UNED). Profesor Titular en la Universidad Central de Venezuela, Universidad Pedagógica Experimental Libertador y Universidad Católica Andrés Bello. Director del Doctorado en Educación (UCAB). Universidad de adscripción: Universidad Católica Andrés Bello (UCAB).

tuliorc1@gmail.com

Orcid ID: https://orcid.org/0000-0002-9012-8707 